\title{
AUTOCUIDADO PARA PESSOAS COM DEFICIÊNCIA ADOUIRIDA: REFLEXÃO SOBRE INTERVENÇÕES DE ENFERMAGEM FRENTE AOS ENFRENTAMENTOS DA REABILITAÇÃO
}

Wiliam César Alves Machado1 Hilmara Ferreira da Silval Wanderley Gurgel de Almeida ${ }^{2}$ Nébia Maria Almeida de Figueiredo ${ }^{1}$ Maria Manuela Martins ${ }^{3}$ Fernando Manuel Dias Henriques ${ }^{4}$ Adriana Dutra Tholl ${ }^{5}$

\begin{abstract}
https://orcid.org/0000-0002-2880-0144 https://orcid.org/0000-0001-5442-8561 https://orcid.org/0000-0002-8044-5186 http://orcid.org/0000-0003-0880-687X https://orcid.org/0000-0003-1527-9940 https://orcid.org/0000-0002-9919-4313 https://orcid.org/0000-0002-5084-9972
\end{abstract}

Objetivo:discutir a aplicação da Teoria do Déficit de Autocuidado na assistência de enfermagem para pessoas com deficiência adquirida, considerando-as potenciais usuárias do cuidado domiciliar de longo prazo. Metodologia: análise reflexiva a partir de 18 estudos disponíveis nas bases de dados da área, publicados no período de 2009 a 2017, considerando a escassa disponibilidade de publicações sobre a temática. Conclui-se que os enfermeiros recorrem à Teoria para nortear suas intervenções junto aos clientes com deficiência adquirida, sem, contudo, considerar que o processo de reabilitação deve ser pautado em estratégicas habilitadoras para minimização da dependência dessas pessoas para cuidados de longo prazo, extramuros institucionais, em seus domicílios e na comunidade.

Descritores: Enfermagem de Reabilitação; Pessoas com Deficiência; Acessibilidade; Resiliência Psicológica; Autocuidado.

\section{SELF-CARE FOR PEOPLE WITH ACQUIRED DISABILITIES: REFLECTION ON NURSING INTERVENTIONS AND COPING WITH} REHABILITATION.

Objective: to discuss the application of the Self-Care Deficit Theory in nursing care for people with acquired disabilities, considering them potential users of long-term home care. The reflexive analysis came from 18 studies available in the area databases, published from 2009 to 2017, considering the scarce availability of publications on the subject. It is concluded that nurses use the Theory to guide their interventions with clients with acquired disabilities, without considering that the rehabilitation process should be guided by enabling strategies to minimize their dependence on long-term care. institutional extramurals, in their homes and in the community.

Descriptors: Rehabilitation Nursing; Disabled Persons; Accessibility; Resilience Psychological; Self Care.

\section{AUTOCUIDADO PARA PERSONAS CON DISCAPACIDAD ADQUIRIDA: REFLEXIÓN SOBRE INTERVENCIONES DE} ENFERMERÍA FRENTE A LOS ENFRENTAMIENTOS DE LA REHABILITACIÓN.

Objetivo era analizar la aplicación de la Teoría del Déficit de Autocuidado en la atención de enfermería para personas con discapacidades adquiridas, considerándolas usuarios potenciales de la atención domiciliaria a largo plazo. El análisis reflexivo provino de 18 estudios disponibles en las bases de datos delárea, publicados de 2009 a 2017, considerando la escasa disponibilidad de publicaciones sobre el tema. Se concluye que las enfermeras utilizan la Teoría para guiar sus intervenciones con clientes con discapacidades adquiridas, sin considerar que el proceso de rehabilitación debe guiarse por estrategias habilitadoras para minimizar su dependencia de la atención a largo plazo. extramuros institucionales, en sus hogares y en la comunidad.

Descriptores: Enfermería en Rehabilitación; Personas con Discapacidad; Accesibilidad; Resiliencia Psicológica.

'Universidade Federal do Estado do Rio de Janeiro-UNIRIO/RJ.

2Universidade Estadual de Roraima - UERR/RR.

${ }^{3}$ Escola Superior de Enfermagem do Porto-ESEP/Portugal.

${ }^{4}$ Escola Superior de Enfermagem de Coimbra - ESEnfC/Portugal.

5Universidade Federal de Santa Catarina-UFSC/SC.

Autor correspondente: Wiliam César Alves Machado. E-mail: Machado.wilmachado@uol.com.br 


\section{INTRODUÇÃO}

Há mais de 40 anos na formação profissional de enfermeiros, desde a graduação, a programas de pósgraduação, especialização, mestrado e doutorado, ou em participação de inúmeras bancas de avaliação de programas em nível regional, nacional e internacional, temos constatado o quão convictos estão os enfermeiros ao afirmarem que utilizam a Teoria do Déficit de Autocuidado (TDA) nas diversas situações de cuidado com seus clientes ${ }^{(1)}$.

Nesta reflexão, cabe destacar impreterivel que se estabeleça similitude entre os diagnósticos da NANDA Internacional(2), as intervenções de Enfermagem pautadas em referencial teórico que lhes confiram caráter acadêmicocientífico, e os resultados alcançados pelo cliente com deficiência adquirida, considerando seus enfrentamentos em curto, médio e longo prazos ${ }^{(3)}$. Assim, a tríade diagnóstico, intervenções e resultados interage como processo determinante para conferir caráter sistemático ao cuidado e assistência prestada em quaisquer situações de saúde, doença, ou processo de reabilitação(l). Ressalta-se que a deficiência adquirida impõe restrições, dificuldades e dependências de vários níveis para as pessoas por ela acometidas ${ }^{(4)}$, cabendo a cada uma decidir o caminho a trilhar, a maneira de reagir para avançar, reabilitar ou sucumbir, por mais que a Enfermagem de Reabilitação as possam auxiliar na superação dos seus desafios e enfrentamentos ${ }^{(3)}$.

Diante de necessidades de saúde cada vez mais complexas, as habilidades de pensar crítica e criativamente são consideradas essenciais aos enfermeiros ${ }^{(5)}$, em especial, os enfermeiros de reabilitação(1), ao se apresentarem dispostos a desenvolver e descobrir novos conhecimentos que emergem do compartilhamento de experiências do autocuidado junto aos clientes e seus cuidadores ${ }^{(6-7)}$. Um dinâmico processo de co-construção de conhecimento para desenvolver habilidades de pensamento crítico e criativo(5), envolvendo enfermeiros, clientes e familiares no autocuidado de longo prazo e domiciliar(3).

Isto posto, como pesquisadores atuantes na área de Enfermagem de Reabilitação, propomos este estudo que tem por objetivo discutir a aplicação da TDA na assistência de Enfermagem para pessoas com deficiência adquirida, considerando-as potenciais usuárias do cuidado domiciliar de longo prazo.

O referencial teórico utilizado foi a TDA de Orem $^{(8)}$, a qual pressupõe que o cuidado é próprio da ação positiva que tem uma prática e um caminho terapêutico, visando manter a vida e o funcionamento normal do ser humano. Orem aborda o autocuidado como prática das ações que os indivíduos iniciam e executam por si mesmos para manter, promover, recuperar e/ou conviver com os efeitos e limitações dessas alterações de saúde, contribuindo assim para sua integridade, funcionamento e desenvolvimento ${ }^{(9-10)}$

Cabe destacar a ideia central na TDA de Orem(8), definida como a capacidade das pessoas em exercitar o autocuidado para manter a vida, a saúde e o bem-estar. Uma habilidade complexa adquirida ao longo da vida, baseada em comportamentos repetidos diariamente e influenciada por aspectos culturais e antecedentes, habilidades e limitações pessoais, experiências de vida, status de saúde e recursos disponíveis ${ }^{(9,11-13)}$.

Orem nos propõe três subteorias que ajudam a compreender a complexidade da assistência à pessoa com deficiência, iniciando-se por descrever o porquê e como as pessoas cuidam de si próprias (Teoria do Autocuidado), segue-se a descrição e explicação das razões pela quais as pessoas podem ser ajudadas através da Enfermagem e a última, as estratégias de intervenção dos enfermeiros (Teoria dos Sistemas de Enfermagem) que colaboram à compreensão de que, para cada autocuidado, o enfermeiro pode recorrer a estratégias diferentes ${ }^{(13)}$

Diante do diagnóstico de Enfermagem Síndrome PósTrauma - Código 00141, o enfermeiro de reabilitação precisa usar de muita criatividade para identificar estratégias de cuidado calcadas na motivação, tornando-se fundamental ouvir e dar feedback ajustado às expectativas da pessoa com deficiência, mas com uma presença positiva, pois o cliente se apresenta recluso, mergulhado em seus labirintos mentais, medos e enfrentamentos emocionais característicos da fase de negação(1).

Como a intervenção precoce é essencial para que os clientes sejam cuidados e orientados pelos enfermeiros na presença de cuidador familiar, disposição para participar e interesse de aprender influenciam os resultados das intervenções da equipe de reabilitação(3). Contudo, muitos clientes são admitidos nos programas de reabilitação sem que tenham superado as fases de negação e repercussão, apresentando-se deveras desmotivados e estagnados no diagnóstico Resiliência Prejudicada - Código 00210(2).

A resiliência é compreendida como a capacidade humana de enfrentar as adversidades, proporcionando ao indivíduo ser transformado por esses fatores potencialmente estressores, adaptando-se ou superando tais experiências traumáticas e/ou estressantes ${ }^{(1,3,4)}$. Caracteriza-se pela capacidade de enfrentar as fases de reabilitação, seus medos, angústias, sensação de perdas, para que emerjam suas potencialidades para se conhecer. A resiliência é discutida não apenas como um atributo inato ou adquirido, mas um processo interativo e multifatorial, envolvendo aspectos individuais, o contexto ambiental, a quantidade e qualidade dos eventos vitais, e a presença dos fatores de promoção do autocuidado. 
As equipes atuantes nos programas institucionalizados de reabilitação, inclusive os enfermeiros, acolhem, cuidam, observam e acompanham atentamente os progressos individuais de elaboração dos enfrentamentos, superações, e o despertar da motivação da pessoa com deficiência adquirida para sua necessidade/responsabilidade de avançar no programa.

O interesse da pessoa com deficiência adquirida em se perceber necessitando de ajuda para cuidar de si, em si, sinaliza que tenha deixado para trás as fases de negação, repercussão e ajustamento, e esteja pronta para adentrar na fase de reconstrução. Momento propício para que o enfermeiro de reabilitação invista nos diagnósticos de Mobilidade Física Prejudicada - Código 00085; e Disposição para Melhora do Autocuidado - Código 00182(2), cujas estratégias de orientação e treinamentos devem envolver os clientes e familiares.

Figura 1. Fluxograma da relação entre Diagnóstico, Intervenções pautadas no Déficit de Autocuidado e Resultados no âmbito do Cuidado de Enfermagem de Reabilitação.

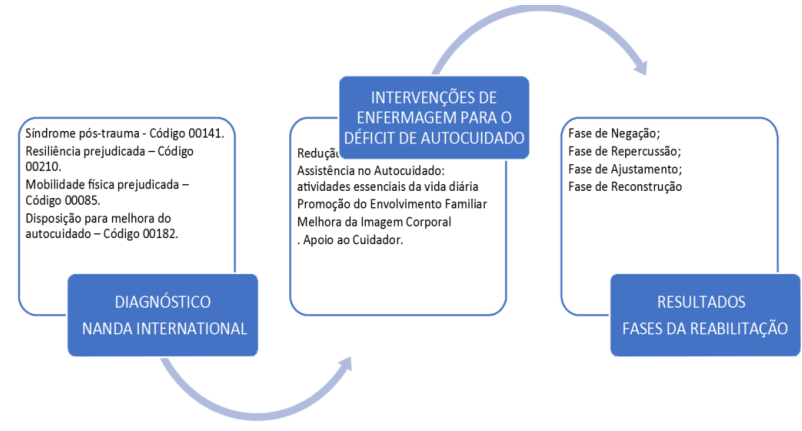

Considerando a carência de publicações que abordem a temática "cuidado de Enfermagem para com pessoas com deficiência adquirida" (grifo dos autores), procedeu-se a seleção dos artigos disponiveis nas bases de dados da área, publicados no período de 2009 a 2017.

A busca das publicações foi realizada em janeiro de 2019, a partir dos artigos de pesquisadores brasileiros publicados na Biblioteca Virtual em Saúde (BVS), no índice bibliográfico da Literatura Latino-Americana e do Caribe em Ciências da Saúde (LILACS), e na Scientific Electronic Library Online (SciELO). Foram definidos os descritores contidos no Medical Subject Headings (MeSH), coincidentes com os pertencentes aos Descritores em Ciências da Saúde (DECS) pesquisados em inglês na BVS: Rehabilitation Nursing. Disabled Persons, Accessibility, Resilience Psychological and Self Care, e português: Enfermagem de Reabilitação; Pessoas com Deficiência; Acessibilidade; Resiliência Psicológica; Autocuidado. Os achados foram contextualizados com a literatura internacional, a partir de consulta ao site da PubMed (National Library of Medicine National Institutes of Health), com base nos mesmos descritores em inglês acima citados.

Assim sendo, por questões de objetividade da presente reflexão e coerência com as normas de publicação do periódico, seguem nove publicações nas quais enfermeiros discorrem sobre suas intervenções junto aos clientes com deficiência adquirida, envolvendo sequelas e enfrentamentos da deficiência física e visual.

Quadro 1: Relações entre tipos de deficiência adquirida, fases da reabilitação e intervenções de enfermagem focadas no déficit de autocuidado: dando voz aos enfermeiros.

\begin{tabular}{|c|c|c|c|}
\hline Publicação & $\begin{array}{c}\text { Tipo de } \\
\text { Deficiência }\end{array}$ & $\begin{array}{c}\text { Fases da } \\
\text { Reabilitação }\end{array}$ & $\begin{array}{l}\text { Abordagens dos } \\
\text { enfermeiros }\end{array}$ \\
\hline $\begin{array}{l}\text { Lessmann, } \\
\text { Conto, } \\
\text { Ramos et al. } \\
2011^{(10)}\end{array}$ & $\begin{array}{l}\text { Fisica } \\
\text { AVC }\end{array}$ & AJ, RC. & $\begin{array}{l}\text { Cuidado de en- } \\
\text { fermagem para o } \\
\text { controle postural, } \\
\text { para os exercicios } \\
\text { de amplitude do } \\
\text { movimento, no } \\
\text { treino de marcha, } \\
\text { nos exercicios } \\
\text { de manutenção } \\
\text { de equilibrio; e } \\
\text { para atividades } \\
\text { cotidianas. }\end{array}$ \\
\hline $\begin{array}{l}\text { Assis, Faro. } \\
2011 .{ }^{(15)}\end{array}$ & $\begin{array}{l}\text { Física } \\
\text { Paraplegia e } \\
\text { Tetraplegia }\end{array}$ & $A J, R C$. & $\begin{array}{l}\text { Treinamento e } \\
\text { motivação para } \\
\text { a técnica catete- } \\
\text { rismos vesicais. } \\
\text { Avaliação do } \\
\text { diário miccional e } \\
\text { das adaptações } \\
\text { realizadas. }\end{array}$ \\
\hline $\begin{array}{l}\text { Souza, } \\
\text { Moura, Nas- } \\
\text { cimento et } \\
\text { al. } 2012(9)\end{array}$ & Visual & NG, RP, AJ, RC. & $\begin{array}{l}\text { Solidão, falta } \\
\text { de interação, } \\
\text { dificuldades } \\
\text { alimentação, } \\
\text { prevenção riscos } \\
\text { de doenças. Inte- } \\
\text { ração social pre- } \\
\text { judicada; déficit } \\
\text { no autocuidado; } \\
\text { risco de sindrome } \\
\text { do estresse } \\
\text { por mudança; } \\
\text { disfunção sexual; } \\
\text { e mobilidade física } \\
\text { prejudicada. }\end{array}$ \\
\hline $\begin{array}{l}\text { Campoy, } \\
\text { Rabeh, } \\
\text { Nogueira et } \\
\text { al. 2012(16) }\end{array}$ & $\begin{array}{l}\text { Física } \\
\text { Paraplegia e } \\
\text { Tetraplégia }\end{array}$ & NG, RP, AJ, RC. & $\begin{array}{l}\text { Solidão, falta } \\
\text { de interação, } \\
\text { dificuldades } \\
\text { alimentação, } \\
\text { prevenção riscos } \\
\text { de doenças. Inte- } \\
\text { ração social pre- } \\
\text { judicada; déficit } \\
\text { no autocuidado; } \\
\text { risco de síndrome } \\
\text { do estresse } \\
\text { por mudança; } \\
\text { disfunção sexual; } \\
\text { e mobilidade física } \\
\text { prejudicada. }\end{array}$ \\
\hline
\end{tabular}




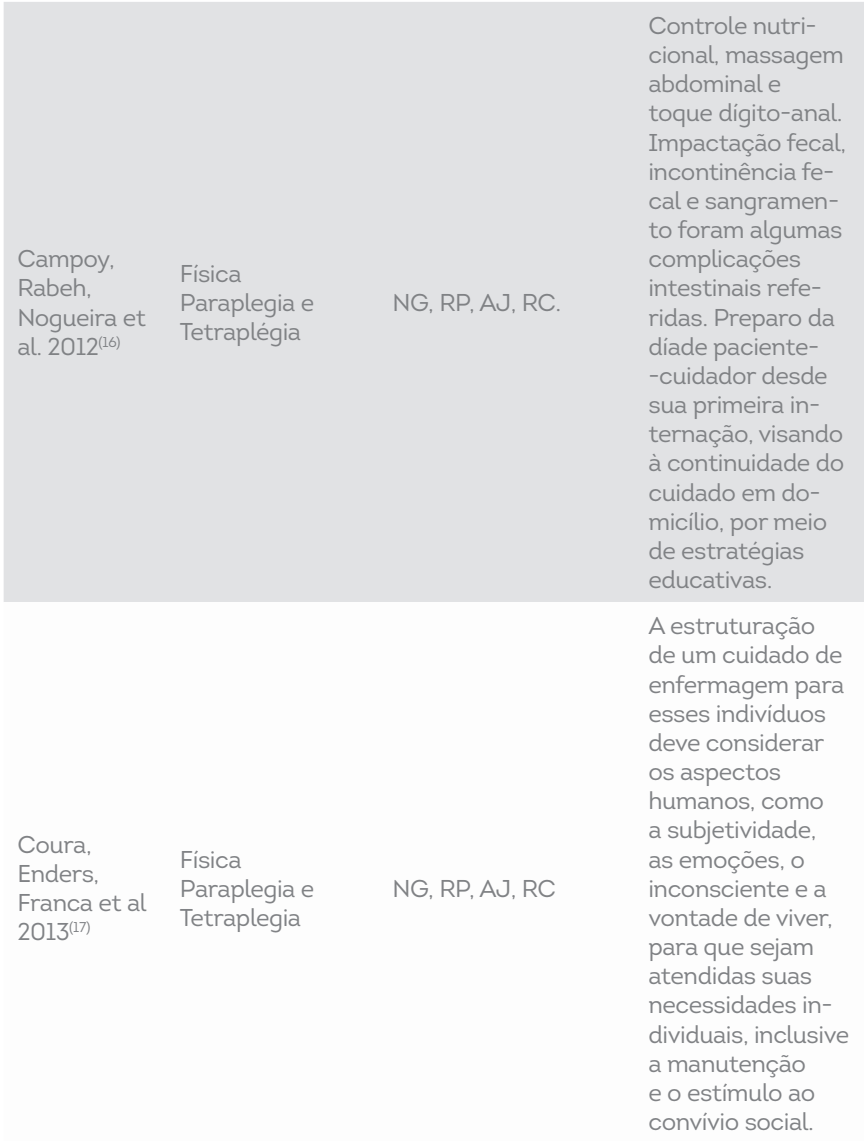

$\begin{array}{lll} & \text { A maioria precisa } \\ & \text { de um tempo para } \\ & \text { incorporar aquele } \\ & \text { novo equipamen- } \\ & \text { to, aquela nova } \\ \text { Ardigo, } & \text { condição. Depois, } \\ \text { Amante. } & \text { Estomia } & \text { com o passar dos } \\ 2013^{(18)} & \text { dias, nós conse- } & \\ & & \text { guimos fazer que } \\ & \text { a pessoa mexa } \\ & \text { [ENF8] }\end{array}$

A Limitação para realização de atividades diárias apresentou associação significativa com equipamento

Silva,

$\begin{array}{lll}\text { Andrade, } & \text { Física } & \text { AJ, RC. } \\ \text { Luz et al. } & \text { Estomia }\end{array}$

$2017^{(19)}$ coletor, que acar-

reta restrições

à vida cotidiana, principalmente limitações na sexualidade, vida social, atividades diárias, vestuário e alimentação.

$\begin{array}{lll} & & \text { A capacidade } \\ & \text { da realização do } \\ & \text { autocuidado deve } \\ \text { Andrade, } & \text { ser avaliada, a } \\ \text { Martins, } & \text { partir do desen- } \\ \text { Medeiros et } & \text { volvimento de } \\ \text { al. } 2017^{(20)} & \text { RP, AJ, RC. } & \text { habilidades do } \\ & \text { paciente e do } \\ & \text { familiar. }\end{array}$

Mauricio

Souza,
Costa et al. Estomia RP, AJ, RC.

$2017^{(21)}$

Importante é o

incentivo à auto-

nomia, à indepen-

dência por meio

do ensino para o

autocuidado. (E 6).

Eles precisam sair

daqui com todas

suas dúvidas

solucionadas

para conseguirem

realizar o cuidado

em seu domicilio

(E 2).

Legendas: Fases da deficiência: Negação = NG; Repercussão = $\mathrm{RP} ;$ Ajustamento $=\mathrm{AJ} ;$ Reconstrução $=\mathrm{RC}$.

Conforme destacado no Quadro 1, as orientações para o autocuidado de clientes com sequela de AVC, paraplégicos, tetraplégicos e ostomizados, são desenvolvidas sem considerar as questões relativas às fases de negação e repercussão(10,15,19), quando eles enfrentam suas vulnerabilidades mentais e emocionais, os autores priorizam apenas os aspectos físicos das limitações funcionais $e$ disfunções nas eliminações impostas aos clientes. Os dois primeiros estudos foram realizados na Região Sul do Brasil com resultados similares aos evidenciados em estudo realizado em 2015, com 50.912 pessoas com sequelas de AVC, na China ${ }^{(22)}$, e com estudo realizado em 2016, com 50 clientes atendidos num centro de reabilitação de cuidados terciários da Arábia Saudita ${ }^{(23)}$. enquanto o terceiro, realizado em 2013, no Piauí, com 96 pessoas.

Ainda no âmbito das orientações dos enfermeiros para o autocuidado dos clientes ostomizados, como destacado no Quadro l, são desenvolvidas sem considerar as vulnerabilidades dessas pessoas na fase de negação $(20,21)$. os autores se debruçam em aspectos operacionais das orientações dos clientes e familiares para o autocuidado. inclusive na garantia de inclusão social e apoio profissional extramuros institucionais.

Da mesma forma, procedeu-se a seleção aleatória dos artigos de pesquisadores brasileiros, disponiveis nas bases de dados da área, publicados no período de 2009 à 2017, estudos pautados em depoimentos dos clientes. Assim sendo, no Quadro 2, seguem nove publicações de pesquisadores brasileiros, nas quais os clientes narram suas experiências de receptores dos cuidados prestados pela equipe de enfermagem visando prepará-los para o autocuidado domiciliar, envolvendo sequelas e seus enfrentamentos na deficiência adquirida modalidades física e auditiva. 
Quadro 2: Relações entre tipos de deficiência adquirida, fases da reabilitação e intervenções de enfermagem focadas no autocuidado: dando voz aos clientes.

\begin{tabular}{|c|c|c|c|}
\hline Publicação & $\begin{array}{c}\text { Tipo de } \\
\text { Deficiência }\end{array}$ & $\begin{array}{c}\text { Fases da } \\
\text { Reabilitação }\end{array}$ & $\begin{array}{l}\text { Relatos dos } \\
\text { clientes }\end{array}$ \\
\hline $\begin{array}{l}\text { Silva, Vas- } \\
\text { concelos, } \\
\text { Santana et } \\
\text { al. } 2010^{(24)}\end{array}$ & $\begin{array}{l}\text { Fisica } \\
\text { Mastectomia }\end{array}$ & NG, RP, AJ & $\begin{array}{l}\text { Sinto-me enver- } \\
\text { gonhada de me } \\
\text { olhar no espelho. } \\
\text { (El6) Eu me sinto } \\
\text { triste hoje, porque } \\
\text { tenho só um seio. } \\
\text { (E6) Porque eu } \\
\text { me vejo mutilada, } \\
\text { um monstro. (E5) }\end{array}$ \\
\hline $\begin{array}{l}\text { Machado, } \\
\text { Scramin. } \\
2010^{(25)}\end{array}$ & $\begin{array}{l}\text { Física } \\
\text { Tetraplegia }\end{array}$ & NG, RP, AJ, RC & $\begin{array}{l}\text { Tenho medo. Por } \\
\text { que se eu ficar } \\
\text { sozinho, quem vai } \\
\text { cuidar das minhas } \\
\text { coisas? (VÊNUS, } \\
45 \text { ANOS) Um } \\
\text { medo que eu } \\
\text { tenho é se morrer } \\
\text { alguém de quem } \\
\text { eu dependo. Esse } \\
\text { é um medo, de } \\
\text { ficar só. (JÚPTER, } \\
25 \text { ANOS). }\end{array}$ \\
\hline $\begin{array}{l}\text { Carvalho, } \\
\text { Santos, } \\
\text { Linhares. } \\
2012^{(26)}\end{array}$ & $\begin{array}{l}\text { Física } \\
\text { Mastectomia }\end{array}$ & NG, RP, AJ, RC & $\begin{array}{l}\text { Enfrentar a } \\
\text { realidade da vida } \\
\text { que chegou, sei } \\
\text { que é ruim. (Dália) } \\
\text { Não fazer esforço } \\
\text { com o braço. (Or- } \\
\text { quidea) Precisa, } \\
\text { assim, de uma } \\
\text { orientação mais } \\
\text { profunda, mais } \\
\text { clara. (Orquidea). }\end{array}$ \\
\hline $\begin{array}{l}\text { Poletto, Sil- } \\
\text { va. } 2013^{(27)}\end{array}$ & $\begin{array}{l}\text { Física } \\
\text { Estomia }\end{array}$ & NG, RP. & $\begin{array}{l}\text { O que eu vou } \\
\text { fazer com isso } \\
\text { aqui (aponta para } \\
\text { o estoma)? (PEl). } \\
\text { Uma enfermeira } \\
\text { foi, fez e mostrou } \\
\text { para minha filha } \\
\text { como que seria } \\
\text { feita a limpeza. Ai } \\
\text { ela fez ali perto } \\
\text { da minha filha e } \\
\text { mostrou é assim, } \\
\text { assim que funcio- } \\
\text { na. Dai em diante } \\
\text { a responsabilida- } \\
\text { de é tua. Mostrou } \\
\text { uma vez. (PE4). }\end{array}$ \\
\hline $\begin{array}{l}\text { Mota, } \\
\text { Gomes, } \\
\text { Petuco et } \\
\text { al. } 2015^{(28)}\end{array}$ & $\begin{array}{l}\text { Fisica } \\
\text { Estomia }\end{array}$ & NG, RP, AJ, RC. & $\begin{array}{l}\text { Adaptei-me } \\
\text { porque queria } \\
\text { viver. (P3). Se a } \\
\text { pessoa não tiver } \\
\text { cabeça boa, não } \\
\text { vai se adaptar e } \\
\text { se cuidar sozinho! } \\
\text { (P9). No Serviço } \\
\text { de Estomaterapia, } \\
\text { a gente tem o } \\
\text { apoio, os profis- } \\
\text { sionais estarão lá } \\
\text { para nos auxiliar } \\
\text { sempre. (P20). }\end{array}$ \\
\hline
\end{tabular}

Minha vida mudou muito [...] não sabia a extensão da gravidade. (El) A vida tem sido terrivel. Péssima. A minha vida se transformou num caos total. Tristeza, sinto raiva, por ter acontecido isso comigo. Eu não me cuido mais. (E3). Curativo e banho, eu faço sozinho, me cuido sozinho. É uma aprendizagem uma lição que a gente cumpre ela sem reclamar. (E6)

$\begin{array}{ll} & \text { É minha mãe me } \\ & \text { dar remédio e eu } \\ & \text { tomar. [Sujeito A]. } \\ \text { Solia, Silva. Auditiva } & \text { Mamãe que cuida } \\ 2017^{(12)} & \text { de mim. Eu faço } \\ & \text { o que ela manda. } \\ & \text { [Sujeito D]. }\end{array}$

O atendimento, o ensinamento que eles passaram para nós foi muito bom. (E3). Aqui

Freire, Angelin, Souza, Física Brandão et Estomia al. $2017^{(29)}$ no hospital não foi suficiente, não (ElO).Acho que a enfermagem deveria ensinar um pouco mais, pois minha filha (cuidadora) não sabe direitinho. (E4)

\begin{tabular}{|c|c|c|c|}
\hline $\begin{array}{l}\text { Dias, Muniz, } \\
\text { Viegas et al. } \\
2017^{(30)}\end{array}$ & $\begin{array}{l}\text { Física } \\
\text { Mastectomia }\end{array}$ & NG, RP, AJ. & $\begin{array}{l}\text { O corpo fica dife- } \\
\text { rente. Não tenho } \\
\text { mais a habilidade } \\
\text { que eu tinha, de } \\
\text { jeito nenhum. Eu } \\
\text { fiquei com muita } \\
\text { depressão, E tu } \\
\text { não tem vontade } \\
\text { às vezes de } \\
\text { levantar, não tem } \\
\text { vontade de tomar } \\
\text { banho. (P4). A } \\
\text { dificuldade ficou } \\
\text { para tudo. Agora } \\
\text { eu consigo tomar } \\
\text { banho sozinha. } \\
\text { (P6). }\end{array}$ \\
\hline
\end{tabular}

Legendas: Fases da deficiência: Negação = NG; Repercussão = RP; Ajustamento = AJ; Reconstrução = RC

O Quadro 2 mostra relevantes relatos dos clientes sobre vulnerabilidades e seus enfrentamentos, como mastectomizadas que superaram fases de negação, repercussão e ajustamento, mesmo passando por vergonha, tristeza e baixa-estima ${ }^{(24)}$; tetraplégicos que superaram as quatro fases da reabilitação, não obstante experimentarem medo de ficar só, e temerem ficar sem apoio e cuidados da família(25). Da mesma forma, clientes mastectomizadas 
demonstram ter superado as quatro fases da reabilitação(26), inclusive, chamando atenção para que as estratégias de orientações para o autocuidade envolvam também seus esposos.

Ainda, conforme relato de clientes ostomizados no Quadro

2, ficou evidente o quanto as estratégias de orientação para o autocuidado influenciam a capacidade de superação das fases da reabilitação. Destaque para relatos de superação da fase de negação e repercussão com ênfase na rejeição da bolsa, nada obstante a pressa dos enfermeiros e indisposição para eliminar dúvidas ${ }^{(27)}$; ̀̀ satisfação e insatisfação quanto aos procedimentos de orientação para o cuidar de si, quando os clientes ostomizados do estudo sugerem ter evoluído e superado as fases de negação, repercussão e ajustamento(29). Culminando em estudo que sugere terem os clientes ostomizados superado as quatro fases da reabilitação(28), relatando algo como "Adaptei-me porque queria viver" ou "Se a pessoa não tiver cabeça boa, não vai se adaptar", mesmo que tenham afirmações relacionadas ao nojo de portar bolsa "cheia de cocô".

Achados similares nos Estados Unidos, com 87 ostomizados(31), e com resultados do estudo na Cidade do Cairo, Egito, em 2015, com 96 clientes ostomizados ${ }^{(32)}$.

Estudos com clientes paraplégicos demonstram ter superado as quatro fases da reabilitação(6), embora tenham enfrentado tristeza, raiva e julgada péssima a vida após a lesão medular, reações características de pessoas jovens que levam mais tempo para elaborar as limitações impostas pela deficiência adquirida. Tanto quanto expressas vulnerabilidades em relatos de mulheres mastectomizadas ${ }^{(30)}$, alegando depressão e dificuldade para tudo, as quais superaram as fases de negação, repercussão e ajustamento, permanecendo sem adentar na fase de reconstrução. Chamou atenção estudo com adolescentes surdos ${ }^{(12)}$, pois implica na dificuldade de comunicação com a equipe para expressar o que de fato sentem em relação à condição de pessoa com deficiência, sugerindo dificuldades referentes à barreira comunicacional e ajustamento aos cuidados dos familiares.

De acordo com a convicção dos enfermeiros sobre como aplicam a TDA para nortear suas intervenções no cuidar de pessoas com deficiência adquirida, percebemse algumas dificuldades no âmbito da compreensão dos seus pressupostos essenciais. O que implica enfatizar que o cuidado é próprio da ação positiva que tem uma prática e um caminho terapêutico, visando manter a vida e o funcionamento normal do ser humano. Em se tratando de pessoas com deficiência adquirida, essas ações positivas devem contemplar necessariamente abordagens focadas no cuidar de si, adequadas as fases de reabilitação enfrentadas por essas pessoas, habilitando-as para o cuidado de longo $\operatorname{prazo}^{(33,34)}$

\section{CONSIDERAÇÕES FINAIS}

É preciso conhecer as abordagens teóricas para que possamos aplicá-las nos contextos de prática profissional da melhor forma possivel. A complexidade da vida, saúde, doença e os processos de reabilitação das pessoas com deficiência adquirida, têm características gerais e singulares. Sendo as primeiras, passiveis de enquadramentos em perspectivas teóricas genéricas, enquanto as outras, carecem de estratégias de intervenção criativas, diretivas, exclusivas, particulares, para se preservar privacidades e vulnerabilidades das pessoas.

O cuidado deve ser documentado com base em sistemas de informação classificados, contudo, isso não impede que os valores do conhecimento específico de Enfermagem sejam aplicados no substrato da intervenção dos enfermeiros. No caso dessa reflexão, adequadas se apresentam as estratégias de orientações sobre o cuidar de si, considerando a capacidade das pessoas para a superação da deficiência adquirida, suas potenciais necessidades de treinamentos para o desenvolvimento de habilidades do cuidado domiciliar, fundamentais para tornar a vida mais autônoma, saudável e inclusiva.

\section{Limitações do estudo}

Considerando o limitado número de estudos realizados e publicados no Brasil sobre o tema desta reflexão, assomada a tendência de foco em determinados procedimentos terapêuticos e tipos de deficiência, outros estudos deverão ampliar a identificação e análise do cuidado e assistência de enfermagem pautada no autocuidado.

Contribuições para a prática profissional de Enfermagem

Os resultados apontados pela reflexão sugerem que a utilização da TDA pelos enfermeiros para com pessoas com deficiência carece de maior diálogo entre diagnóstico, intervenção e resultados, como ferramentas determinantes para que o cuidado domiciliar de longo prazo, executado pelos seus familiares e cuidadores, consolidando a visibilidade e reconhecimento do enfermeiro no contexto da sociedade contemporânea.

\section{Contribuição dos autores}

WCAM, concepção, desenho, análise e interpretação dos dados, redação do artigo, revisão crítica, revisão final. NMAF, MMM, análise e interpretação dos dados, redação do artigo, revisão crítica. ADT, FMDH, WGA, SSB e HFS redação do artigo, revisão crítica, revisão final. 


\section{REFERÊNCIAS}

1. Figueiredo NMA, Machado WCA, Martins MM. REABILITAÇÃO: nômades em busca de sentido para o cuidado da pessoa com deficiência adquirida. Curitiba: CRV; 2018. 308 p.

2. NANDA International. Diagnósticos de enfermagem da NANDA: definições e classificações 2018-2020. Porto Alegre: Artmed; 2018. 364 p.

3. Machado, WCA. O cotidiano na perspectiva da pessoa com deficiência. Curitiba: CRV; 2017. 138 p.

4. Freire LIF, Fernandes C. A base de conhecimentos dos professores, a reflexão e o desenvolvimento profissional: um estudo de caso a partir da escrita de diários de aula por estagiários de professores de Quimica. Rev. Bras. Estud. Pedagog. [Internet]. 2015 [cited 2019 Jan 09]: 96(243): 359-79. Available from: http://www. scielo.br/pdf/rbeped/ v96n243/2176-6681-rbeped-96-243-00359.pdf

5. Vargas CP. Diaz PS, Menegaz JC, Backes VMS, Kempfer SS, Lima DKS. Introduction of the Flipped Classroom in nursing teaching. Rev. enferm. UFSM. [Internet]. 2018 [cited 2019 Jan 13]; 8(4): 829-40. Available from: https://periodicos.ufsm.br/reufsm/article/view/26811/pdf 6. Alvarez, AB, Machado, WCA, Teixeira, MLO., Castelo Branco, E, Figueiredo NMA. Body image in paraplegics: coping with changes from the perspective of people with spinal cord injury. Rev. enferm. UERJ. [Internet]. 2016 [cited 2019 Jan 18]; 24(1): el6125. Available from: http://www.e-publicacoes.uerj.br/index.php/enfermagemuerj/article/ view/16125

7. Machado WCA, Silva VM da, Silva RA da, Ramos RL, Figueiredo NMA de, Branco EMSC, Rezende LK, Carreiro MA. Hospital discharge of patients with disabling neurological injury: necessary referrals to rehabilitation. Ciênc. Saúde Colet. [Internet]. 2016 [cited 2019 Jan 07];

21(10): 3161-70. Available from: http://www.scielo.br/scielo.php?scrip-

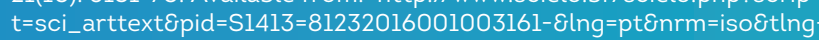
en

8. Orem DE. Nursing concepts of practice. Saint Louis (US): $6^{\mathrm{a}} \mathrm{ed}$. Mosby. 2001. $158 p$

9. Souza ELV de: Moura GN de: Nascimento JC do: Lima MA de: Pagliuca LMF; Caetano JA. Nursing diagnoses based on the self-care theory in people with visual deficiency. Rev Rene [Internet]. 2012 [cited 2019 Jan 18]; 13(3): 542-51. Available from: http://periodicos.ufc.br/rene/ article/view/3975/3139

10. Lessmann JC, Conto FD, Ramos G, Borenstein MS, Meirelles BHS. Nursing activities in self-care and rehabilitation of patients who suffered Stroke. Rev. bras. enferm. [Internet]. 2011 [cited 2019 Jan 29]; 64(1): 198-202. Disponivel em: http://www.scielo.br/scielo.php?scrip$\mathrm{t}=\mathrm{sci} \_$arttextEpid=S0034-71672011000100030\&lng=pt\&nrm=iso 11. Stacciarini TSG, Pace AE. Confirmatory factor analysis of the Appraisal of Self-Care Agency Scale - Revised. Rev. latinoam. enferm. [Internet]. 2017 [cited 2019 Jan 15]; 25: e2856. Available from: http:// www.scielo.br/pdf/rlae/v25/0104-1169-rlae-25-02856.pdf 12. Solia FSF, Silva SS. Health education through dialogue processes, and the self-care of the deaf individual. Ciênc. educ. (Bauru). [Internet]. 2017 [cited 2019 Jan 14]: 23(3): 677-89. Disponivel em: http://www.scielo.br/scielo.php?script=sci_arttext\&pi$d=$ S1516-73132017000300677\&lng=pt\&nrm=iso

13. Quiros PJP, Vidina TSS, Almeida Filho AJ. Self-care: Orem's theoretical contribution to the Nursing discipline and profession. Referência. [Internet]. 2014 [cited 2019 Jan 21]; serIV(3): 157-164. Available from: http://www.scielo.mec.pt/scielo.php?script=sci_arttextEpi$\mathrm{d}=$ S0874-02832014000300018\&lng=es\&nrm=iso\&tlng=pt

14. Ferreira CL, Santos LMO, Maia EMC. Resilience among the elderly cared for by the Primary Healthcare Network in a city of Northeast Brazil. Rev. Esc. Enferm. USP. [Internet]. 2012 [cited 2019 Jan 22]; 46(2): 328-34. Available from http://www.scielo.br/scielo.php?script=sci_arttextEpi$d=S 0080-62342012000200009 \& \operatorname{lng}=$ pt\&nrm=iso\&tlng=en 15. Assis GM, Faro ACM. Clean intermittent self catheterization in spinal cord injury. Rev. Esc. Enferm. USP. [Internet]. 2011 [cited 2019 Jan 19]; 45(1): 289-93. Available from: http://www.scielo.br/pdf/reeusp/ v45nl/en_4l.pdf

16. Campoy LT, Rabeh SAN, Nogueira PC, Vianna PC, Miyazaki MY.
Self-care practice for bowel functioning in a group of patients with spinal cord injury. Acta fisiátrica. [Internet]. 2012 [cited 2019 Jan 14] 19(4): Available from: http://www.actafisiatrica.org.br/detalhe_artigo. asp?id $=482$

17. Coura AS, Enders B, ISX, Vieira CENK, Dantas DNA, Menezes DJC. Ability for self-care and its association with sociodemographic factors of people with spinal cord injury. Rev. Esc. Enferm. USP. [Internet]. 2013 [cited 2019 Jan 06]; 47(5): 1150-57. Available from: http://www.scielo.br/scielo.php?script=sci_arttextEpi$\mathrm{d}=50080-62342013000501150 \& \operatorname{lng}=\mathrm{pt}$ \&nrm=iso

18. Ardigo FS, Amante LN. Knowledge of the professional about nursing care of people with ostomies and their families. Texto Contexto Enferm. [Internet]. 2013 [cited 2019 Jan 29]; 22(4): 1064-71. Available from: http://www.scielo.br/pdf/tce/v22n4/en_24.pdf

19. Silva CRDT, Andrade EMLR, Luz MHBA, Andrade JX, Silva GRF. Quality of life of people with intestinal stomas.Acta Paul. Enferm. [Internet]. 2017 [cited 2019 Jan 29]; 30(2): 144-51. Available from: http:// www.scielo.br/pdf/ape/v30n2/en_1982-0194-ape-30-02-0144.pdf

20. Andrade RS, Martins JM, Medeiros LP, Souza AJG, Torres GV, Costa IKF. Sociodemographic, clinical and self-care aspects of persons with intestinal stoma. Rev. enferm. UERJ. [Internet]. 2017 [cited 2019 Jan 26]; 25:e19368. Available from: https://www.e-publicacoes.uerj.br/ index.php/enfermagemuerj/article/view/19368/24239

21. Mauricio VC, Souza NDO, Costa CCP et al. The view of nurses about educational practices targeted at people with a stoma. Esc. Anna Nery [Internet]. 2017 [cited 2019 Jan 12]; 21(4): e20170003. Disponivel em: http://www.revenf.bvs.br/pdf/ean/v2ln4/1414-8145ean-2177-9465-EAN-2017-0003.pdf

22. Wen T, Liu B, Wan X, Zhang X, Zhou X, Lau AYL, Zhang Y. Risk factors associated with 31-day unplaned readmission in 50,912 discharged patients after stroke in China. BMC Neurol. [Internet]. 2018 [cited 2019 Jan 22]: 18(1): 218. Available from: https://bmcneurol.biomedcentral.com/articles/10.1186/s12883-018-1209-y

23. AlSaleh AJ, Qureshi AZ, Syamsuddin Abdin Z, Mushabbab AlHabter A. Long-term compliance with bladder management in patients with spinal cord injury: A Saudi-Arabian perspective. J Spinal Cord Med. [Internet]. 2018 [cited 2019 Jan 16]: 22:1-6. Available from: https:// www.tandfonline.com/doi/abs/10.1080/10790268.2 018.1531609?journalCode=yscm 20

24. Silva SED, Vasconcelos EV, Santana ME, Rodrigues ILA, Leite TV et al. Social representations of women submitted to mastectomy and the implications for self-care. Rev. bras. enferm. [Internet]. 2010 [cited 2019 Jan 08]; 63(5): 727-34. Available from: http://www.scielo. br/scielo.php?script=sci_arttext\&pid=S0034-71672010000500006 25. Machado WCA, Scramin AP. Functional (in)dependence in the dependent relationship of quadriplegic men with their (un)replaceable parents/caregivers. Rev. Esc Enferm. USP. [Internet], 2010 [cited 2019 Jan 06]; 44(1): 53-60. Available from: http://www.scielo.br/scielo.php?script=sci_arttextEpi$d=50080-62342010000100008 \& \operatorname{lng}=p t \& n r m=i s o \& t$ lng $=e n$

26. Carvalho APR de, Santos TMB dos, Linhares FMP. Promotion of self-care for mastectomized women. Cogitare enferm. [Internet]. 2012 [cited 2019 Jan 15]; 17(3): 485-491. Available from: https://revistas.ufpr.br/cogitare/article/view/29290/19039

27. Poletto D. Silva DMGV. Living with intestinal stoma: the construction of autonomy for care. Rev. latinoam. enferm. [Internet]. 2013 [cited 2019 Jan 09]: 21(2): 531-538. Available from: http://www.scielo. $\mathrm{br} / \mathrm{pdf} / \mathrm{rlae} / \mathrm{v} 21 \mathrm{n} 2 / 0104-1169-\mathrm{rlae}-21-02-0531 . p d f$

28. Mota MS, Gomes GC, Petuco VM. Heck RM, Barros EJL, Gomes VLO. Facilitators of the transition process for the self-care of the person with stoma: subsidies for Nursing. Rev. Esc. Enferm. USP. [Internet]. 2015 [cited 2019 Jan 22]:. 49(1): 82-8. Available from: http:// www.scielo.br/pdf/reeusp/v49nl/0080-6234-reeusp-49-01-0082.pdf 29. Freire DA, Angelin RCM, Souza NR, Brandão BMGM et al. Self-image and self-care in the experience of ostomy patients: the nursing look. REME rev. min. enferm. [Internet]. 2017 [cited 2019 Jan 26]: 21 : el019. Available from: http://www.reme.org.br/artigo/detalhes/1155

30. Dias LV. Muniz RM, Viegas AC et al. Mastectomized woman in 
31. Ercolano E, Grant M, McCorkle R et al. Applying the Chronic Care Model to Support Ostomy Self-Management: Implications for Oncology Nursing Practice. Clin J Oncol Nurs. [Internet]. 2016 [cited 2019 Jan 18]; 20(3): 269-74. Available from: https://www.ncbi.nlm.nih.gov/ pmc/articles/PMC5544017/

32. Boraii S. A Descriptive Study to Assess Quality of Life in Egyptian Patients With a Stoma. Ostomy Wound Manage. [Internet]. 2017 [cited 2019 Jan 16]; 63(7): 28-33. Available from: https://www.o-wm.com/article/descriptive-study-assess-quality-life-egyptian-patients-stoma 33. Machado WCA, Pereira JS, Schoeller SD, Júlio LC, Martins MM, Figueiredo NMA. Comprehensiveness in the care network regarding the care of the disabled person. Texto Contexto Enferm. [Internet].
2018 [cited 2019 Jan 14]; 27(3): e4480016. Available from: http://www.scielo.br/pdf/tce/v27n3/en_0104-0707-tce-2703-e4480016.pdf

34 - Leite MS, Aguiar LC. Diagnósticos de enfermagem em pacientes submetidos à colostomia. Enferm Foco. [Internet]. 2017 [cited 2019 Jan 21]; 8(2): 72-6. Avsailable from: http://revista.cofen.gov.br/index php/enfermagem/article/view/1227 